\title{
Dietary phosphate exacerbates intestinal inflammation in experimental colitis
}

\author{
Kohei Sugihara, ${ }^{1}$ Masashi Masuda, ${ }^{1}$ Mari Nakao, ${ }^{1}$ Maerjianghan Abuduli, ${ }^{1}$ Yukiko Imi, ${ }^{1}$ Naoko Oda, ${ }^{1}$ \\ Toshiya Okahisa, ${ }^{2}$ Hironori Yamamoto, ${ }^{3}$ Eiji Takeda ${ }^{1}$ and Yutaka Taketani ${ }^{1}$,* \\ 'Departments of Clinical Nutrition and Food Management and 2Department of Gastroenterology and Oncology, Institute of Biomedical Sciences, \\ Tokushima University Graduate School, Tokushima 770-8503, Japan \\ ${ }^{3}$ Department of Health and Nutrition, Faculty of Human Life, Jin-ai University, Fukui 915-8586, Japan
}

(Received 9 December, 2016; Accepted 26 December, 2016; Published online 28 July, 2017)

\begin{abstract}
The recent widespread consumption of Western diets and food additives worldwide is associated with excessive inorganic phosphate intake. However, researchers have known little about the impact of dietary phosphate intake on the development of inflammatory bowel disease to date. In this study, we investigated the effects of dietary phosphate on intestinal inflammation in experimental colitis. Sprague-Dawley rats were fed different phosphate diets $(0.5 \%, 1.0 \%$ and $1.5 \%$ phosphate) with or without dextran sulfate sodium. For in vitro study, the effects of phosphate on proinflammatory cytokine induction and reactive oxygen species production in RAW264.7 macrophage were examined. Dietary phosphate exacerbated intestinal inflammation in experimental colitis in a dose-dependent manner, as assessed by the clinical disease activity score, colon length, and histology. Furthermore, the high phosphate diet increased myeloperoxidase activity and proinflammatory cytokine mRNA expression through the activation of nuclear factor $\kappa B$ in the inflamed colon. In addition, high phosphate loading in RAW264.7 cells directly enhanced reactive oxygen species production and proinflammatory cytokine gene expression. Our results demonstrated that the high phosphate diet exacerbated intestinal inflammation in experimental colitis. These findings have important therapeutic implications for inflammatory bowel disease patients.
\end{abstract}

Key Words: dietary Pi, DSS-induced colitis, proinflammatory cytokine, NF-kB, ROS

I nflammatory bowel disease (IBD) is a term describing a group of chronic recurrent inflammatory disorders of the gastrointestinal tract, typified by Crohn's disease and ulcerative colitis. The exact pathogenesis of IBD is complex and involves genetic factors, the host immune system, and environmental factors. ${ }^{(1)}$ With environmental factors, the variety of nutrients is a major factor affecting the intestinal inflammation and microbiota, and diet has been implicated in the development of these diseases. $^{(2)}$ Several diets - such as high intake of refined sugarcontaining food, fast foods, and red and processed meat-have been identified as risk factors for the onset and relapse of IBD. ${ }^{(3-5)}$ In particular, fast foods and processed meat contain high fat levels, which results in exacerbation of intestinal inflammation, but these foods also frequently contain a large amount of inorganic phosphate $(\mathrm{Pi})$ as a food additive.

$\mathrm{Pi}$ is an essential nutrient for skeletal formation, energy metabolism, and intracellular signaling, and is mainly supplied from the diet including meat, grains, and dairy products. ${ }^{(6)}$ Furthermore, $\mathrm{Pi}$ is the main component of many food additives which are utilized for various purposes. Although there is little clear evidence to shows increasing $\mathrm{Pi}$ intake in the general population, excessive consumption and widespread use of $\mathrm{Pi}$ is currently raise a public health concern. ${ }^{(7,8)}$ Indeed, recent epidemiological studies have suggested that high dietary $\mathrm{Pi}$ intake is associated with increased cardiovascular risk and all-cause mortality..$^{(9,10)}$ Moreover, high Pi loading in the body can cause a wide range of cellular and tissue injuries. ${ }^{(11-15)}$ This Pi cytotoxicity due to excessive retention of $\mathrm{Pi}$ was shown to be associated with increased mortality and cardiovascular complication in patients with chronic kidney disease (CKD). ${ }^{(16,17)}$ Thus, dietary $\mathrm{Pi}$ restriction and $\mathrm{Pi}$ binder administration are beneficial treatments for CKD patients to prevent the progression of diseases and cardiovascular complications.

Along with other researchers, we recently reported that $\mathrm{Pi}$ metabolism was altered by lipopolysaccharide (LPS)-induced systemic inflammation. ${ }^{(18)}$ whereas $\mathrm{Pi}$ overloading activated nuclear factor $\kappa \mathrm{B}(\mathrm{NF}-\kappa \mathrm{B})$ signaling via reactive oxygen species (ROS) production, ${ }^{(15)}$ thus suggesting an interactive association between Pi and inflammation. These interactions between serum Pi levels and serum inflammatory markers are also observed in CKD patients. ${ }^{(19)}$ Furthermore, low-Pi diet and Pi binder treatment ameliorated systemic inflammation in CKD model rat, ${ }^{(14)}$ and thus excess Pi intake was linked to inflammation; itself an important potential mediator of inflammatory disease.

Increasing evidence from both clinical and animal studies suggests that Western diets aggravate intestinal inflammation. However, several reviews of dietary management in IBD patients did not mention dietary Pi intake, and little is known about the impact of dietary Pi on intestinal inflammation.

In this study, we examined the effects of dietary Pi on intestinal inflammation using an experimental colitis rat model. We demonstrated that high Pi diet exacerbated intestinal inflammation in experimental colitis, and Pi loading directly enhanced ROS production and proinflammatory cytokines production via $\mathrm{NF}-\kappa \mathrm{B}$ activation in RAW264.7 macrophages.

\section{Materials and Methods}

Animal and experimental design. Seven-week-old male Sprague-Dawley rats were purchased from Japan SLC (Shizuoka, Japan) and individually caged in a climate-controlled room $\left(22 \pm 2{ }^{\circ} \mathrm{C}\right)$ with a 12-h light:12-h dark cycle. All rats were randomly divided into 6 groups and fed different Pi diets $(0.5 \%$, $1.0 \%$ and $1.5 \% \mathrm{Pi}$ ) with or without dextran sulfate sodium (DSS). In this study, the $0.5 \% \mathrm{Pi}$ diet was designated as the control diet according to the previous studies. ${ }^{(20,21)}$ The diets were based on modified AIN-93G rodent diet, formulated with casein and $\mathrm{K}_{2} \mathrm{HPO}_{4}$ (Table 1). Rats were fed different Pi diets for 7 days before DSS treatment, and then given $2.5 \%$ DSS [molecular weight

*To whom correspondence should be addressed. E-mail: taketani@tokushima-u.ac.jp 
Table 1. Composition of the experimental diets

\begin{tabular}{lccc}
\hline & Pi: $0.5 \%(\mathrm{~g})$ & $\mathrm{Pi}: 1.0 \%(\mathrm{~g})$ & $\mathrm{Pi}: 1.5 \%(\mathrm{~g})$ \\
\hline AIN-93G & 59.5 & 59.5 & 59.5 \\
Milk casein & 20 & 20 & 20 \\
Sugar & 4.77 & 4.77 & 4.77 \\
Mineral Mix & 1.56 & 1.56 & 1.56 \\
$\mathrm{CaCO}_{3}$ & 1.26 & 1.26 & 1.26 \\
Soy bean oil & 7 & 7 & 7 \\
$\mathrm{KH}_{2} \mathrm{PO}_{4}$ & 1.52 & 3.71 & 5.91 \\
$\mathrm{KCl}_{\text {Dextrin }}$ & 2.41 & 1.2 & 0 \\
Total & 1.99 & 0.99 & 0 \\
\hline
\end{tabular}

We used a mineral mix and an altered AIN-93G diet without casein, $\mathrm{CaCO}_{3}$, and $\mathrm{KH}_{2} \mathrm{PO}_{4}$.

(MW): 36,000-50,000; MP Biomedicals, Illkirch-Graffenstaden, France] orally in drinking water for 7 days. Changes in body weight and characteristics of stool were observed during the DSS treatment period, and the rats were then euthanized under anesthesia at day 7 for evaluation of colitis severity. The disease activity index (DAI) score was assessed using the method described previously. ${ }^{(22)}$ Distal segments of colon were sampled for histological evaluation, and adjacent segments of colon were taken for analysis of gene and protein expression and myeloperoxidase (MPO) activity. The experiments were approved by the Animal Care and Use Committee of Tokushima University and were performed in accordance with the guidelines for the care and handling of laboratory animals.

Histological analysis. Colonic tissues were fixed with $4 \%$ paraformaldehyde, embedded in paraffin, and cut into slices $5 \mu \mathrm{m}$ thick. Colonic sections were deparaffinized, rehydrated, and stained with hematoxylin and eosin for routine histology, or with Alcian blue and periodic acid-Schiff (AB/PAS). All histological quantitation was performed blinded using the scoring system previously described. ${ }^{(23)}$ The scoring system includes severity of inflammation (0-3: none, slight, moderate, severe); extent of injury (0-3: none, mucosal, mucosal and submucosal, transmural); and crypt damage (0-4: none, basal $1 / 3$ damaged, basal $2 / 3$ damaged, only surface epithelium intact, entire crypt and epithelium lost). The score of each score was multiplied by a factor reflecting the percentage of tissue involvement $(\times 1: 0-25 \%, \times 2$ : 26-50\%, $\times 3: 51-75 \%, \times 4: 76-100 \%$ ).

Measurement of myeloperoxidase (MPO) activity. Colonic MPO activity was measured according to the method shown in a previous report. ${ }^{(24)}$ Briefly, colonic tissues were homogenized using a Polytron homogenizer in ice-cold $50 \mathrm{mM}$ potassium phosphate buffers ( $\mathrm{pH} 6.0$ ) containing $0.5 \%$ hexadecyltrimethylammonium bromide. The homogenate was then sonicated for $10 \mathrm{~s}$, freeze-thawed 3 times, and centrifuged at 20,000 $\mathrm{g}$ for $30 \mathrm{~min}$ at $4^{\circ} \mathrm{C}$. The supernatant was added in $50 \mathrm{mmol} / \mathrm{L}$ phosphate buffer (pH 6.0) containing $0.167 \mathrm{mg} / \mathrm{ml} \mathrm{O}$-dianisidine hydrochloride and
$0.0005 \%$ hydrogen peroxide. The kinetics of absorbance at $450 \mathrm{~nm}$ was measured using a spectrophotometer at $25^{\circ} \mathrm{C}$. Protein concentration of the supernatant was determined using a Bradford assay kit (Bio-Rad Laboratories, Hercules, CA) for calibration. MPO was expressed in units per mg of protein; 1 unit of MPO activity was defined as degrading $1 \mu \mathrm{mol}$ hydrogen peroxide/min at $25^{\circ} \mathrm{C}$.

Cell culture and treatment. The RAW264.7 murine macrophage cell line was kindly provided by Prof. T. Nikawa (Tokushima University) and were cultured medium consisted of Dulbecco's modified Eagles medium (DMEM; Sigma-Aldrich, St. Louis, MO) supplemented with $10 \%$ fetal bovine serum (Biowest, Nuaillé, France), $100 \mu \mathrm{g} / \mathrm{ml}$ streptomycin, and $100 \mathrm{U} / \mathrm{ml}$ Penicillin (Sigma-Aldrich). The RAW264.7 cells were grown in $100 \mathrm{~mm}$ tissue culture dishes to $70-80 \%$ confluence and cultured at $37^{\circ} \mathrm{C}$ in a humidified atmosphere containing 5\% $\mathrm{CO}_{2}$. The DMEM contain $0.9 \mathrm{mM}$ phosphate in itself and, RAW264.7 cells were treated with appropriate amounts of sodium phosphate buffer $(0.1 \mathrm{M}$ $\mathrm{Na}_{2} \mathrm{HPO}_{4} / \mathrm{NaH}_{2} \mathrm{PO}_{4}, \mathrm{pH}$ 7.4) to produce final $\mathrm{Pi}$ concentrations.

RNA extraction and real-time reverse transcription polymerase chain reaction (RT-PCR). Total RNA was extracted from colonic mucosa using an RNeasy plus mini kit (Qiagen $\mathrm{GmbH}$, Hilden, Germany) and from RAW264.7 cells using TRIzol Reagent. We purified RNA using the lithium chloride procedure described previously, ${ }^{(25)}$ since DSS is known to interfere with PCR reactions. The cDNA was synthesized using a reverse transcriptase kit (Invitrogen) with an oligo-dT primer. After cDNA synthesis, real-time RT-PCR (Applied Biosystems, Carlsbad, CA) was performed using SYBR Green PCR master mix (Thermo Fisher Scientific, Waltham, MA). The amplification programs were set as follows: initial denaturation at $95^{\circ} \mathrm{C}$ for $10 \mathrm{~min}$, followed by 40 cycles of $95^{\circ} \mathrm{C}$ for $10 \mathrm{~s}, 60^{\circ} \mathrm{C}$ for $15 \mathrm{~s}$, and $72^{\circ} \mathrm{C}$ for $15 \mathrm{~s}$. The primer sequences are shown in Table 2. All sample mRNA levels were normalized to GAPDH for animal or $\beta$-actin for RAW264.7 cells and the relative mRNA levels were calculated.

Nuclear protein extraction and electrophoretic mobility shift assay. Nuclear protein was extracted using buffer A [10 mM HEPES-KOH (pH 7.8), $0.1 \mathrm{mM}$ EDTA, $5 \mathrm{mM} \mathrm{KCl,}$ $0.1 \%$ NP40] containing $0.5 \mathrm{mM}$ PMSF, $1 \mathrm{mM}$ DTT, and protease inhibitor. After centrifugation at $9,100 \mathrm{~g}$ for $1 \mathrm{~min}$ at $4^{\circ} \mathrm{C}$, the supernatant was collected as a cytosol extract. The nuclear pellets were washed 3 times with buffer $\mathrm{A}$ and resuspended in buffer $\mathrm{C}$ [50 mM HEPES-KOH (pH 7.8), 0.1 mM EDTA, $420 \mathrm{mM} \mathrm{KCl,}$ $5 \mathrm{mM} \mathrm{MgCl}_{2}, 20 \%$ glycerol]. After centrifugation at $12,700 \mathrm{~g}$ for 15 min at $4^{\circ} \mathrm{C}$, the supernatant was collected as a nuclear extract. Electrophoretic mobility shift assay (EMSA) analysis was performed as previously described.(26) Briefly, purified DNA fragments were radiolabeled with $\left[\gamma-{ }^{32} \mathrm{P}\right]$ ATP $(110 \mathrm{TBq} / \mathrm{mmol}$; ICN, Costa Mesa, CA) using T4 polynucleotide kinase (Takara, Shiga, Japan). Prepared nuclear protein were incubated with the radiolabeled probe in binding buffer $[10 \mathrm{mM}$ Tris/ $\mathrm{HCl}, \mathrm{pH} 7.5$, $1 \mathrm{mM}$ dithiothreitol, $1 \mathrm{mM}$ EDTA, $10 \%$ glycerol, $1 \mathrm{mM} \mathrm{MgCl}_{2}$, $0.25 \mathrm{mg} / \mathrm{ml} \mathrm{BSA}, 2.5 \mu \mathrm{g} / \mathrm{ml}$ salmon sperm DNA and $2 \mu \mathrm{g}$

Table 2. Sequence of oligonucleotide primers in the quantitative RT-PCR analysis

\begin{tabular}{|c|c|c|}
\hline Gene name & Forward & Reverse \\
\hline rat TNF- $\alpha$ & 5'-GTCGTAGCAAACCACCAAGC-3' & 5'-TGTGGGTGAGGAGCACGTAG-3' \\
\hline rat MCP-1 & 5'-CCCTAAGGACTTCAGCACCTTTG-3' & 5'-AAGTGCTTGAGGTGGTTGTGG-3' \\
\hline rat MUC2 & 5'-CATCAAAGGTGGTGATGTGG-3' & 5'-AGCTGCACGGACACCTCTAT-3' \\
\hline rat GAPDH & 5'-AGTTCAACGGCACAGTCAAG-3' & 5'-GTGGTGAAGACGCCAGTAGA-3' \\
\hline mouse TNF- $\alpha$ & 5'-AGCCTGTAGCCCACGTCGTA-3' & 5'-TCTTTGAGATCCATGCCGTTG-3' \\
\hline mouse IL-1 $\beta$ & 5'-GTGGACCTTCCAGGATGAGG-3' & 5'-CGGAGCCTGTAGTGCAGTTG-3' \\
\hline mouse MCP-1 & 5'-CCCAATGAGTAGGCTGGAGA-3' & 5'-TCTGGACCCATTCCTTCTTG-3' \\
\hline mouse $\beta$-actin & 5'-CTGGGGTGTTGAAGGTCTCAAACATG-3' & 5'-CTGACCCTGAAGTACCCCATTGAACA-3' \\
\hline
\end{tabular}


poly(dI-dC) (dI-dC) (GE Health Care, Tokyo, Japan)] in a final volume of $20 \mu \mathrm{l}$ for $30 \mathrm{~min}$ at room temperature $\left(20^{\circ} \mathrm{C}\right)$. NF- $\kappa \mathrm{B}$ consensus oligonucleotides sequence was purchased from Santa Cruz Biotechnology (Dallas, TX; catalogue number sc-2505). The reaction mixture was then subjected to electrophoresis on a $5 \%$ polyacrylamide gel with $0.1 \times \mathrm{TBE}$ running buffer for $1.5 \mathrm{~h}$ at $150 \mathrm{~V}$. The gel was dried and analyzed with a Bio-imaging analyzer (FLA-9000; GE Health Care).

Immunofluorescence staining. Colonic sections were deparaffinized, rehydrated, and microwaved in $10 \mathrm{mmol} / \mathrm{L}$ sodium citrate ( $\mathrm{pH} \mathrm{6.0)} \mathrm{for} 5 \mathrm{~min}$ to retrieve antigen. After a blocking step with blocking one histo (Nacalai Tesque, Kyoto, Japan) for $10 \mathrm{~min}$ at room temperature, slides were incubated with primary antibodies for NF-אB p65 (1:400, Santa Cruz Biotechnology) overnight at $4^{\circ} \mathrm{C}$. Subsequently, they were washed in phosphatebuffered saline (PBS) and incubated with Alexa Fluor 555conjugated secondary antibodies (Molecular Probes, Eugene, OR) for $1 \mathrm{~h}$ at room temperature. The slides were then counterstained with 4',6-diamidino-2-phenylindole (DAPI; Sigma) to stain nuclei. RAW264.7 cells were cultured on glass cover-slips in 12well plates in vitro. After the indicated treatment, the cells were washed with cold PBS twice, fixed with $4 \%$ paraformaldehyde for $15 \mathrm{~min}$, and permeabilized with $0.1 \%$ Triton X-100 in PBS for $5 \mathrm{~min}$. The cells were then blocked in $0.8 \%$ bovine serum albumin

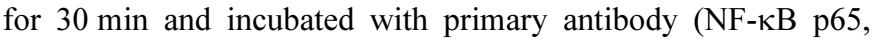
$1: 400)$ for $1 \mathrm{~h}$ at room temperature. After being washed 3 times, secondary antibody-(conjugated with Alexa Fluor 568 and DAPI) was stained for $1 \mathrm{~h}$ at room temperature. Fluorescence was visualized using a BZ-9000 fluorescence microscope (Keyence, Osaka, Japan).

Western blot analysis. The nuclear and cytosol extract were mixed with sample buffer, incubated at $95^{\circ} \mathrm{C}$ for $5 \mathrm{~min}$ in the presence of 2-mercaptoethanol, and subjected to sodium dodecyl sulfate polyacrylamide gel electrophoresis (SDS-PAGE). Following electrophoresis, the proteins were transferred to a polyvinylidene difluoride membrane (Immobilon-P; Merck Millipore, Darmstadt, Germany). The membranes were blocking with $5 \%$ skim milk and treated with primary antibody for NF- $\kappa \mathrm{B}$ p65 $(1: 1,000)$, Lamin B $(1: 1,000$, Santa Cruz Biotechnology), and $\beta$ actin $\left(1: 2,000\right.$, Sigma) overnight at $4^{\circ} \mathrm{C}$. The membranes were then incubated with horseradish peroxidase-conjugated secondary antibody. The blots were visualized with the enhanced chemiluminescence (ECL) reagents.

Measurement of ROS production. We measured ROS production using the aminophenyl fluorescein (APF; Goryo Kagaku, Hokkaido, Japan) indicators, which can specifically recognize many types of ROS. ${ }^{(27)}$ RAW264.7 cells were placed on a 96-well plate in $200 \mu \mathrm{l}$ medium. The following day, cells were rinsed with modified Hank's balanced salt solutions (HBSS) and incubated with $20 \mu \mathrm{M}$ APF (diluted in HBSS) for $30 \mathrm{~min}$ at $37^{\circ} \mathrm{C}$. After incubation with APF, cells were treated by HBSS containing different concentration of $\mathrm{Pi}$ with or without specific oxidase inhibitors for $120 \mathrm{~min}$. Fluorescence intensity at excitation $490 \mathrm{~nm}$ and emission $515 \mathrm{~nm}$ was measured by a fluorescent plate reader.

Statistics. Data are expressed as mean \pm SEM. Statistical analysis was performed using unpaired $t$ test for two-group comparisons and one-way ANOVA with Tukey post-hoc test for multigroup comparison. All data analysis was performed using GraphPad Prism 5 software (Graphpad Software, San Diego, CA). $P<0.05$ was considered to indicate statistical significance.

\section{Results}

Effects of dietary Pi on clinical symptoms in DSS-induced colitis. First, we observed the clinical symptoms including body weight loss and stool consistency and bleeding caused by colitis after DSS administration. All DSS-treated groups developed signs of colitis, such as weight loss and diarrhea during the study period.
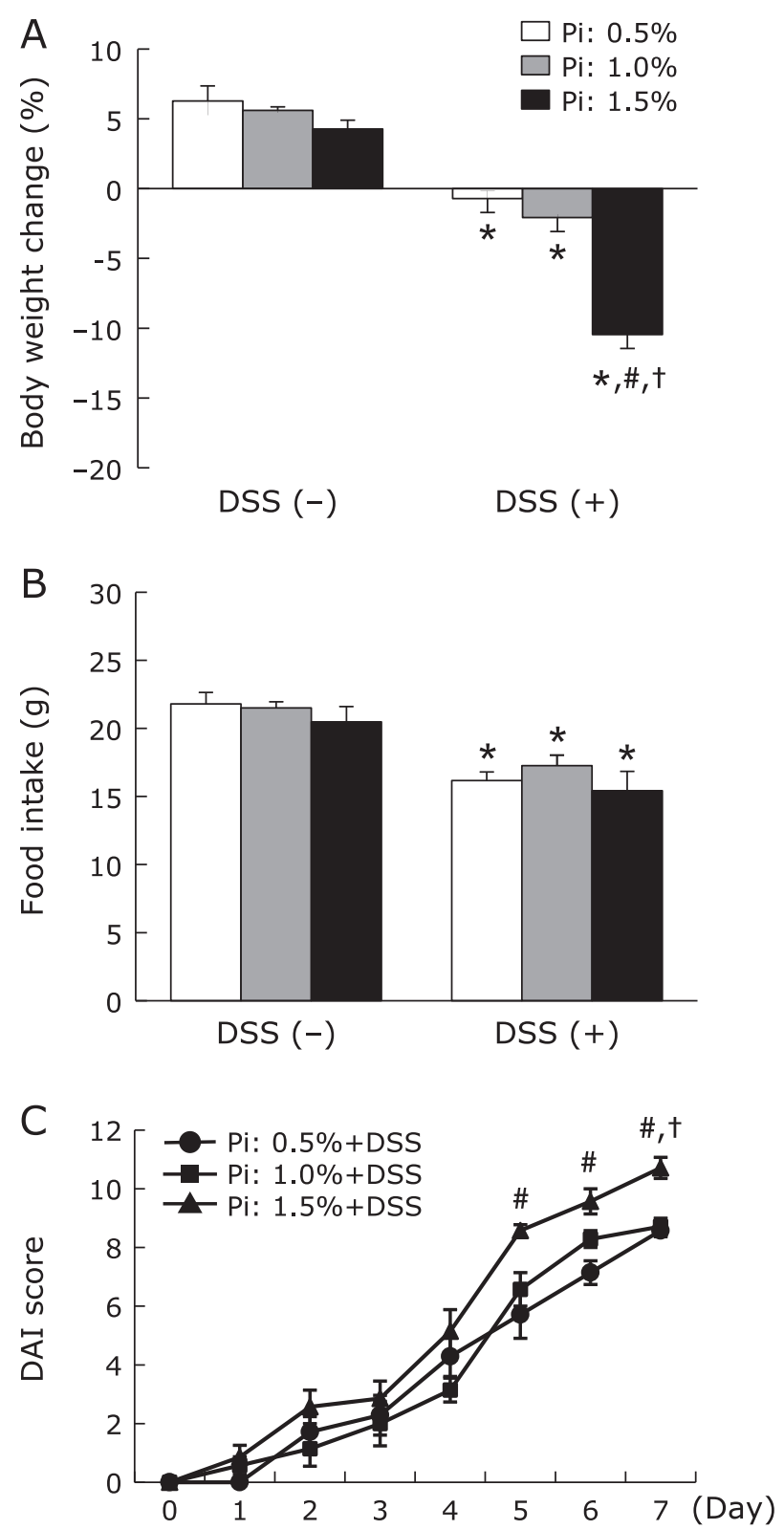

Fig. 1. Increase in dietary $\mathrm{Pi}$ intake exacerbated clinical symptoms in DSS-induced colitis. (A) Changes in body weight during DSS-treated periods. (B) Food intake during DSS-treated periods. (C) Changes in DAl score calculated by summing the means of 3 scores (stool consistency, bleeding, and weight loss) after DSS treatment. Data are expressed as mean \pm SEM $(n=5-7)$. ${ }^{*} p<0.05$ vs control of same dietary Pi group; ${ }^{\#} p<0.05$ vs Pi: $0.5 \%+$ DSS group; ${ }^{\dagger} p<0.05$ vs Pi: $1.0 \%+$ DSS group.

Body weight was significantly decreased in all DSS-treated groups and these changes were more severe in dose-dependent effect of Pi intake (Fig. 1A). Although there was no significant difference in food intake among the different Pi diets, it was significantly lower in the DSS-treated groups compared to the groups not receiving DSS (Fig. 1B). Furthermore, the high Pi diet $(1.5 \%)$ significantly aggravated the DAI score in rats treated with DSS (Fig. 1C).

Effects of dietary Pi on histopathology and mucin expression in DSS-induced colitis. Next, we assessed the severity of DSS-induced colitis macroscopically. As shown in Fig. 2A, the colon length shortened by DSS treatment was more severe in the high Pi diet (1.5\%) groups. The severity of colonic inflammation 

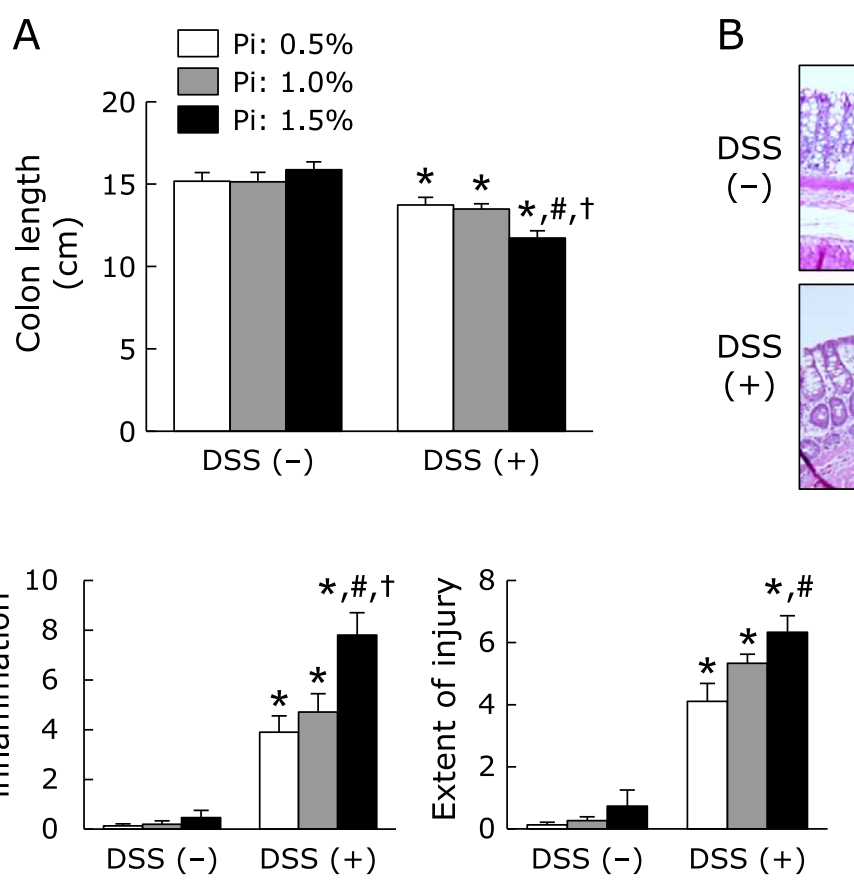

$\mathrm{Pi}: 0.5 \%$
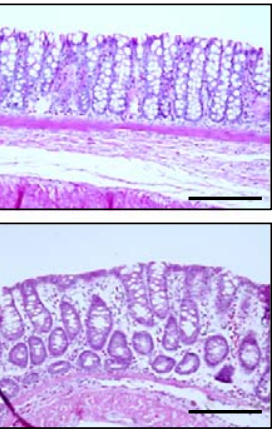

Pi: $1.0 \%$

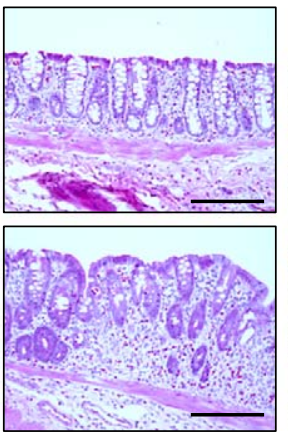

Pi: $1.5 \%$

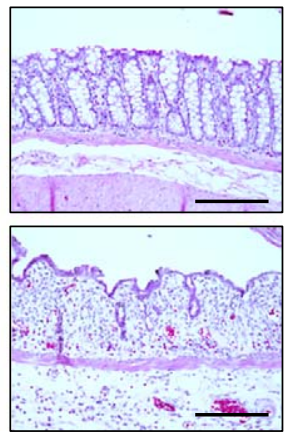

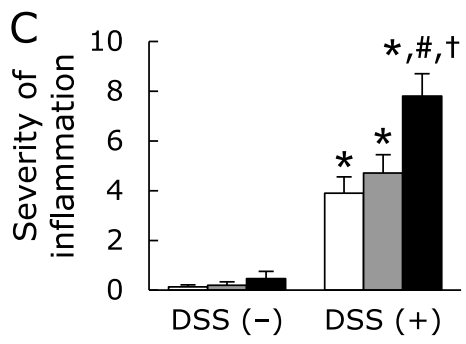
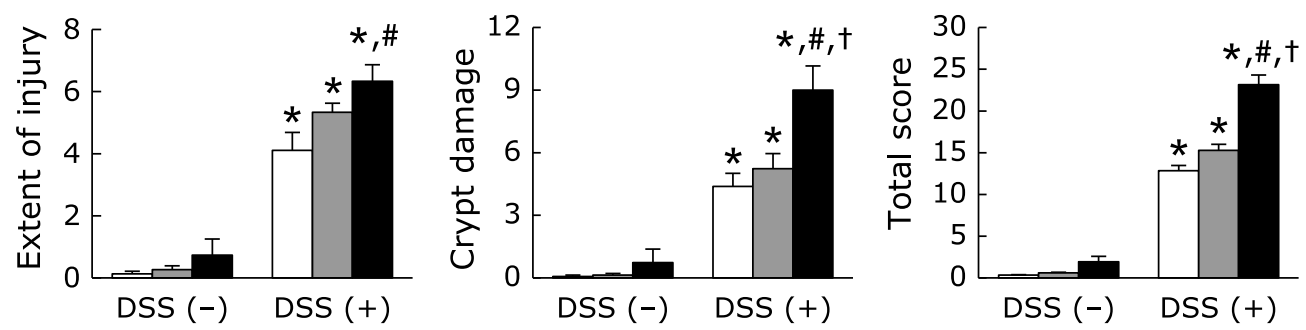

Fig. 2. Increase in dietary Pi intake aggravated histopathology findings in DSS-induced colitis. (A) Changes in colon length. (B) Hematoxylin and eosin staining of colonic tissue. Magnification bar, $200 \mu \mathrm{m}$. (C) Histological scores (severity of inflammation, extent of injury, crypt damage, and total score) after DSS treatment. Data are expressed as mean \pm SEM $(n=5-7)$. ${ }^{*} p<0.05$ vs control of same dietary Pi group; ${ }^{\sharp} p<0.05$ vs Pi: $0.5 \%+$ DSS group; ${ }^{+} p<0.05$ vs Pi: $1.0 \%+$ DSS group.

A
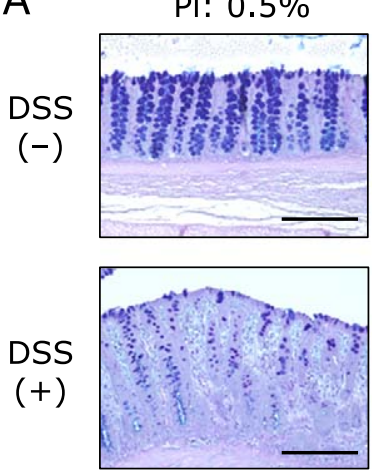

Pi: $1.0 \%$
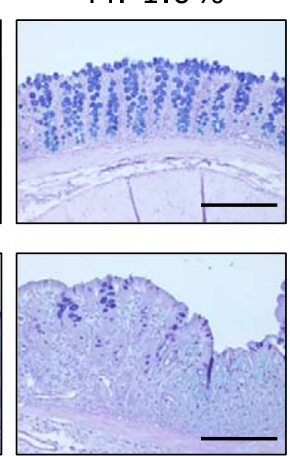

Pi: $1.5 \%$
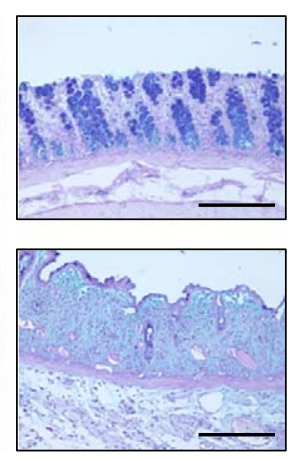
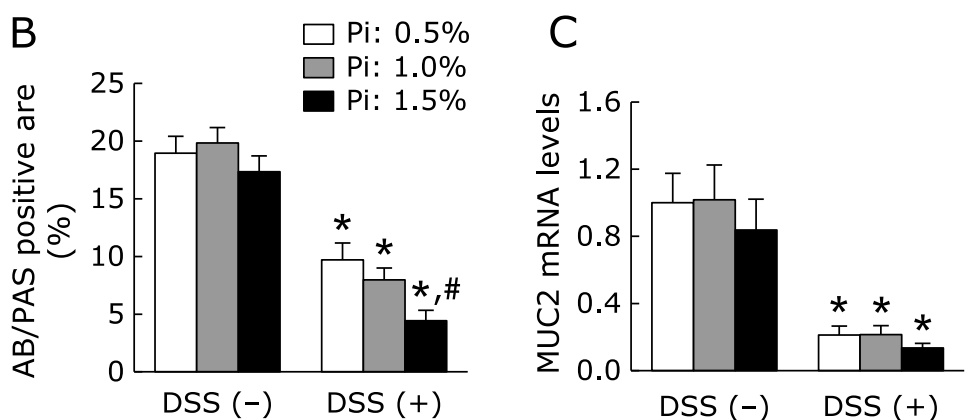

Fig. 3. Effects of dietary Pi on colonic mucus secretion in DSS-induced colitis. (A) AB/PAS staining of colonic tissue in DSS-treated rat. Magnification bar, $200 \mu \mathrm{m}$. (B) Proportion of AB/PAS positive area in the colonic section of DSS-treated rat. Three sections randomly chosen from each rat were analyzed. (C) Mucin 2 (MUC2) mRNA levels of colonic tissue in DSS-induced rats were determined by quantitative real-time RT-PCR analysis. Data are expressed as mean \pm SEM $(n=4-7)$. * $p<0.05$ vs control of same dietary Pi group; ${ }^{*} p<0.05$ vs Pi: $0.5 \%+$ DSS group. 
A

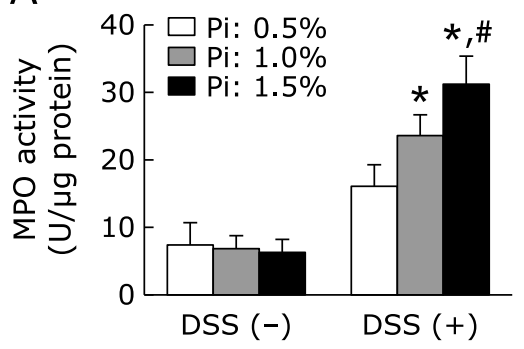

D
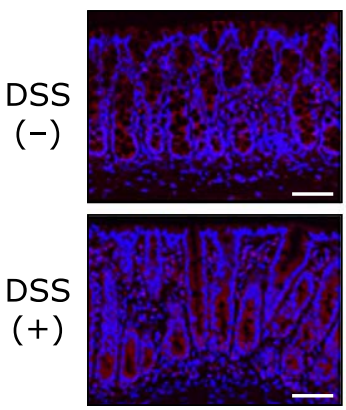

B

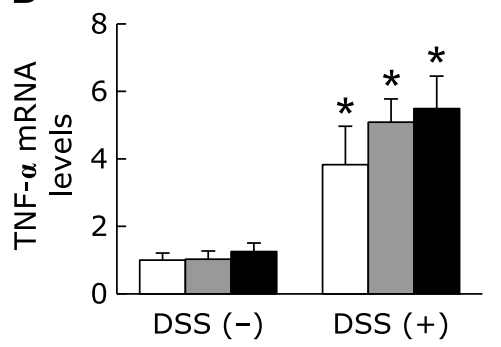

C

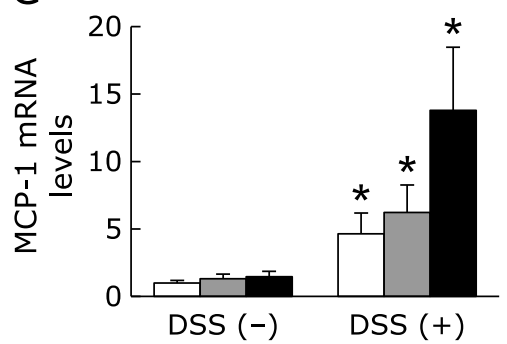

Fig. 4. Increase in dietary $\mathrm{Pi}$ intake enhanced colonic inflammation through the activation of NF- $\mathrm{B}$ in DSS-induced colitis. (A) MPO activity in the colonic tissue of DSS-induced rat. $(B, C)$ Colonic inflammatory cytokine/chemokine mRNA levels in DSS-induced rats were determined by quantitative real-time RT-PCR analysis. (D) NF- $\mathrm{B}$ p65 expression of colonic tissue was analyzed by immunofluorescent staining. Magnification bar, $300 \mu \mathrm{m}$. (E) NF- $\kappa$ B activity of colonic nuclear extract was evaluated by EMSA. Data are expressed as mean \pm SEM ( $n=4-7)$. ${ }^{*} p<0.05$ vs control of same dietary Pi group; ${ }^{\#} p<0.05$ vs $\mathrm{Pi}: 0.5 \%$ + DSS group; ${ }^{\dagger} p<0.05$ vs $\mathrm{Pi}: 1.0 \%+$ DSS group.

was further evaluated by histopathologic observations. As shown in Fig. 2B, DSS treatment induced destruction of epithelial architecture with a loss of crypts and epithelial integrity, submucosal edema, and infiltration of inflammatory cells. In particular, the high $\mathrm{Pi}$ diet $(1.5 \%)$ group had more severe colonic inflammation and epithelial damage. These histologic observations were scored using criteria for histologic scoring of colitis as described above. Dietary Pi significantly exacerbated all histological scores in DSS-treated group in a dose-dependent manner (Fig. 2C). Furthermore, AB/PAS staining identified a significantly reduced number of mucous-secreting goblet cells in DSS-treated groups receiving a high Pi diet, especially $1.5 \%$ Pi group (Fig. 3A and B). Mucin 2 (MUC2) mRNA expression synthesized by the goblet cells in the colon was evaluated. Pi treatment did not affect colonic MUC2 mRNA expression in either the presence or absence of DSS (Fig. 3C).

Effect of dietary $\mathrm{Pi}$ on colonic inflammation in DSSinduced colitis. MPO activity in the colonic tissue was measured to evaluate inflammatory status. MPO activity was significantly higher in DSS treated groups; it was more severe in $\mathrm{Pi}$ intake group in a dose-dependent effect of Pi intake (Fig. 4A). We also measured colonic tumor necrosis factor- $\alpha$ (TNF- $\alpha)$ and monocyte chemottractant protein-1 (MCP-1) mRNA expression, which have been well characterized as a pivotal inflammatory cytokine/chemokine during colonic inflammation. As shown in Fig. 4B and C, TNF- $\alpha$ and MCP-1 mRNA levels were significantly increased in the DSS-treated group and they tended to be higher in the high Pi diet (1.5\%) group in the presence of DSS.

To examine the ability of dietary Pi to stimulate inflammatory cytokine induction through an NF- $\mathrm{KB}-$ mediated pathway, we analyzed NF- $\kappa \mathrm{B}$ expression and activation by immunofluorescence staining and EMSA, respectively. Immunofluorescence staining showed that NF- $\kappa B$ p65 was strongly expressed in intestinal epithelial cells and infiltrating inflammatory cells of inflamed colon in the DSS-treated group, and its expression was increased

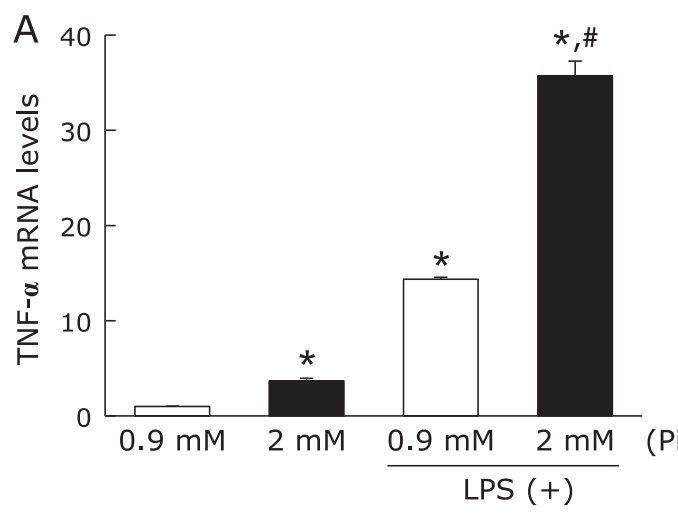

(Pi)

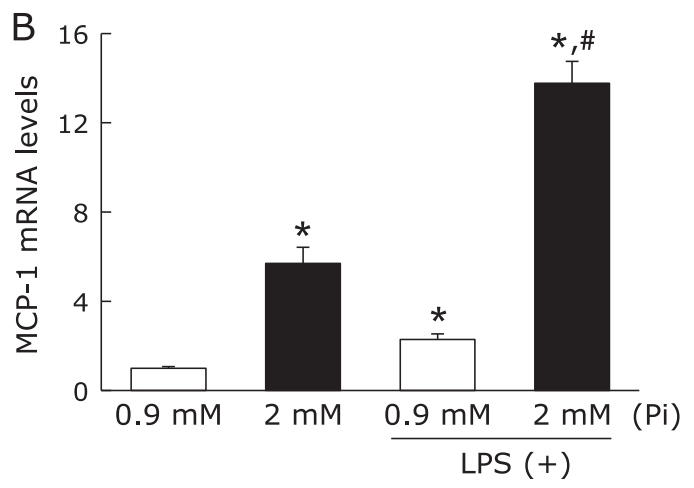

Fig. 5. High Pi loading enhanced inflammatory response in LPSstimulated RAW264.7 cells. RAW264.7 cells were preincubated with Pi $(2 \mathrm{mM})$ for $12 \mathrm{~h}$, followed by an incubation with LPS $(1 \mu \mathrm{g} / \mu \mathrm{l})$ for $1 \mathrm{~h}$. Total RNA was extracted and relative TNF- $\alpha$ (A) and MCP-1 (B) mRNA levels was determined by quantitative real-time RT-PCR. Data are expressed as mean \pm SEM $(n=3) .{ }^{*} p<0.05$ vs Pi: $0.9 \mathrm{mM}$; ${ }^{*} p<0.05$ vs $\mathrm{Pi}: 0.9 \mathrm{mM}+$ LPS. 
in high Pi diet ( $\mathrm{P}: 1.5 \%)$ groups. Consistent with proinflammatory cytokine expression, EMSA analysis demonstrated NF $-\kappa B$ activity was dose-dependently increased in colonic nuclear extracts of DSS-induced colitis rats.

Effect of Pi loading on inflammatory response in RAW264.7 cells. To elucidate the impact of $\mathrm{Pi}$ on the inflammatory response of macrophages, the effects of $\mathrm{Pi}$ on cytokine/chemokine mRNA expression were evaluated by in vitro experiments using RAW264.7 macrophages. First, these cells were incubated with Pi for $12 \mathrm{~h}$, and then exposed to LPS for $1 \mathrm{~h}$. As a result, Pi loading synergistically increased LPS-stimulated TNF- $\alpha$ and MCP-1 mRNA expression in RAW264.7 cells (Fig. 5). Interestingly, in the absence of LPS, treatment of RAW264.7 cells with $\mathrm{Pi}$ significantly increased cytokine/chemokine mRNA expression. Thus, we also examined time- and dose-dependent effects of $\mathrm{Pi}$ on the expression of cytokine/chemokine to determine whether Pi directly causes an inflammatory response in the absence of LPS. RAW264.7 cells were treated with Pi in the absence of LPS for up to $24 \mathrm{~h}$. TNF- $\alpha$ mRNA levels significantly increased within $1 \mathrm{~h}$, and then declined to basal levels at $24 \mathrm{~h}$. Furthermore, the effect of Pi on TNF- $\alpha$ and MCP-1 mRNA expression exhibited dose dependency (Fig. 6A and B), indicated that high Pi loading directly enhanced these cytokine/chemokine gene expressions. Next, we evaluated whether Pi loading directly stimulates proinflammatory cytokine induction through the NF- $\mathrm{BB}-$ mediated pathway. Since the nuclear translocation of the NF- $\mathrm{B}$ p65 is a critical step for the activation of $\mathrm{NF}-\kappa \mathrm{B}$ signaling pathway, we investigated the impact of $\mathrm{Pi}$ on nuclear translocation of the $\mathrm{NF}-\kappa \mathrm{B}$ p65 by immunofluorescence staining and Western blot analysis. As expected, immunofluorescence staining showed that $\mathrm{Pi}$-induced the transfer of NF- $\mathrm{BB}$ p65 into the nucleus, whereas untreated cells did not cause NF- $\kappa \mathrm{B}$ p 65 translocation (Fig. 6C). A similar result was acquired by Western blot analysis with nuclear extracts (Fig. 6D and E). To determine whether NF- $\kappa$ B activation was a prerequisite for proinflammatory cytokine induction, the effect of NF- $\kappa$ B inhibition on RAW264.7 cells was examined. Sulforaphane (SFN), which is an aliphatic isothiocyanate and known anti-inflammatory compound, inhibited LPS-induced NF- $\kappa$ B activation in RAW264.7 cells. ${ }^{(28)}$ SFN pretreatment inhibited Pi-induced TNF- $\alpha$ and MCP-1 mRNA expressions (Fig. 6B). Similarly, Pi-induced nuclear translocation of NF- $\kappa B$ p65 was also suppressed by SFN in RAW264.7 cells (Fig. 6C, D and E). Thus, NF- $\kappa$ B activation was required for the induction of inflammatory cytokine in Pi-stimulated RAW264.7 cells.

Effect of Pi loading on ROS production in RAW264.7 cells. Since the activation of NF- $\kappa \mathrm{B}$ was mediated by ROS, ${ }^{(29)}$ we evaluated whether high Pi loading could enhance ROS production. We determined ROS production using APF, which is a specific fluorescence probe for ROS. Pi loading significantly increased the ROS production in RAW264.7 cells in a dosedependent manner (Fig. 7A). It is reported that Pi-induced ROS is produced mainly by the activation of $\mathrm{NAD}(\mathrm{P}) \mathrm{H}$ oxidase and mitochondrial respiratory chain. Thus, we next examined the effect of specific oxidase inhibitors on Pi-mediated ROS production. Fig. 7A showed that diphenyl iodonium (DPI), which is a specific inhibitor of NAD $(\mathrm{P}) \mathrm{H}$ oxidase, suppressed Pi-induced ROS production. On the other hand, rotenone, an inhibitor of mitochondrial complex I could not inhibit ROS production. Fur-
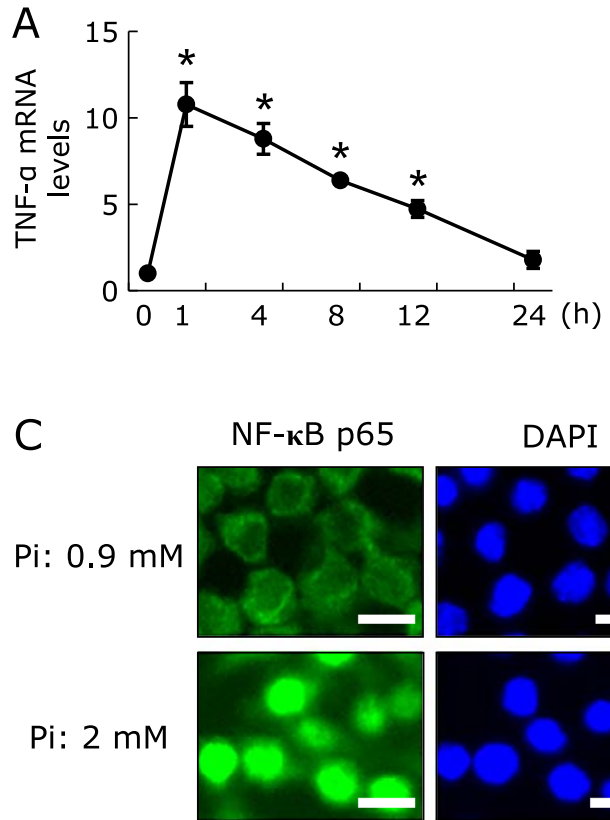

$\mathrm{Pi}: 2 \mathrm{mM}$
+ SFN

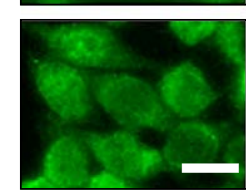

DAPI
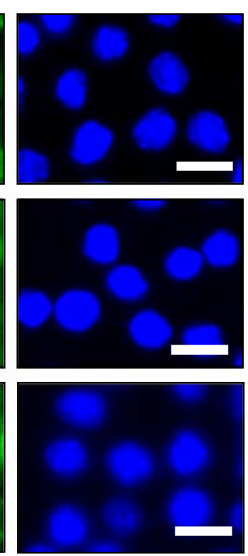

B
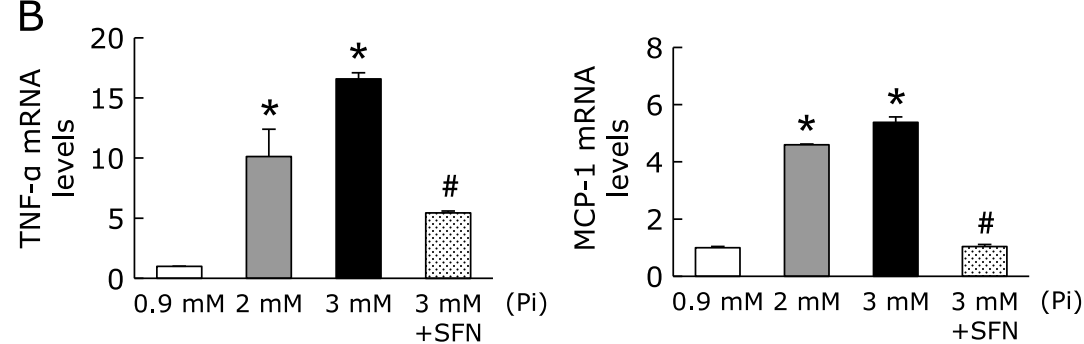

D
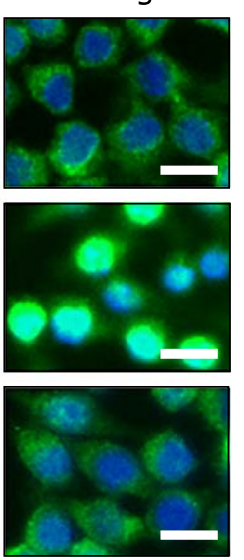

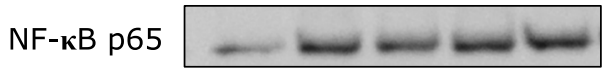

Lamin B

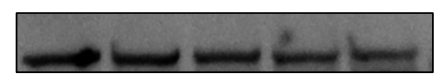

E

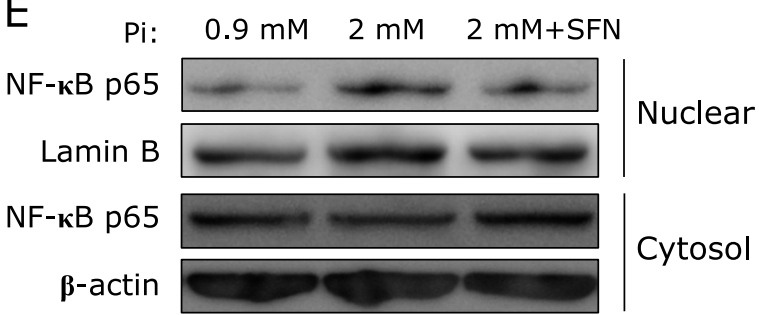

Fig. 6. High Pi loading directly increased cytokine/chemokine mRNA expression through the activation of NF-kB in RAW264.7 cells. (A) RAW264.7 cells were treated with Pi $(2 \mathrm{mM})$ for the indicated times $(n=3)$. (B) RAW264.7 cells were pretreated with SFN $(10 \mu \mathrm{M})$ for $1 \mathrm{~h}$, followed by treatment with different Pi concentrations for $1 \mathrm{~h}(n=3)$. Relative TNF- $\alpha$ and MCP-1 mRNA levels were determined by quantitative real-time RT-PCR. (C) RAW264.7 cells were pretreated with SFN (10 $\mu \mathrm{M})$ for $1 \mathrm{~h}$ and then exposed to Pi $(2 \mathrm{mM})$ for 30 min. Cells were fixed, permeabilized and stained with NF-kB p65 and DAPI. Magnification bar, $10 \mu \mathrm{m}$. (D) RAW264.7 cells were treated with Pi $(2 \mathrm{mM})$ for the indicated times and nuclear protein was extracted. Nuclear NF- $\mathrm{kB}$ p65 expression was determined by Western blot analysis. (E) RAW264.7 cells were pretreated with SFN (10 $\mu \mathrm{M})$ for $1 \mathrm{~h}$, and then treated with Pi $(2 \mathrm{mM})$ for $1 \mathrm{~h}$. Nuclear and cytosol protein was extracted and NF-kB p65 expression was detected by Western blot analysis. Data are expressed as mean \pm SEM. ${ }^{*} p<0.05$ vs Pi: 0.9 mM; ${ }^{*} p<0.05$ vs Pi: 3 mM. 

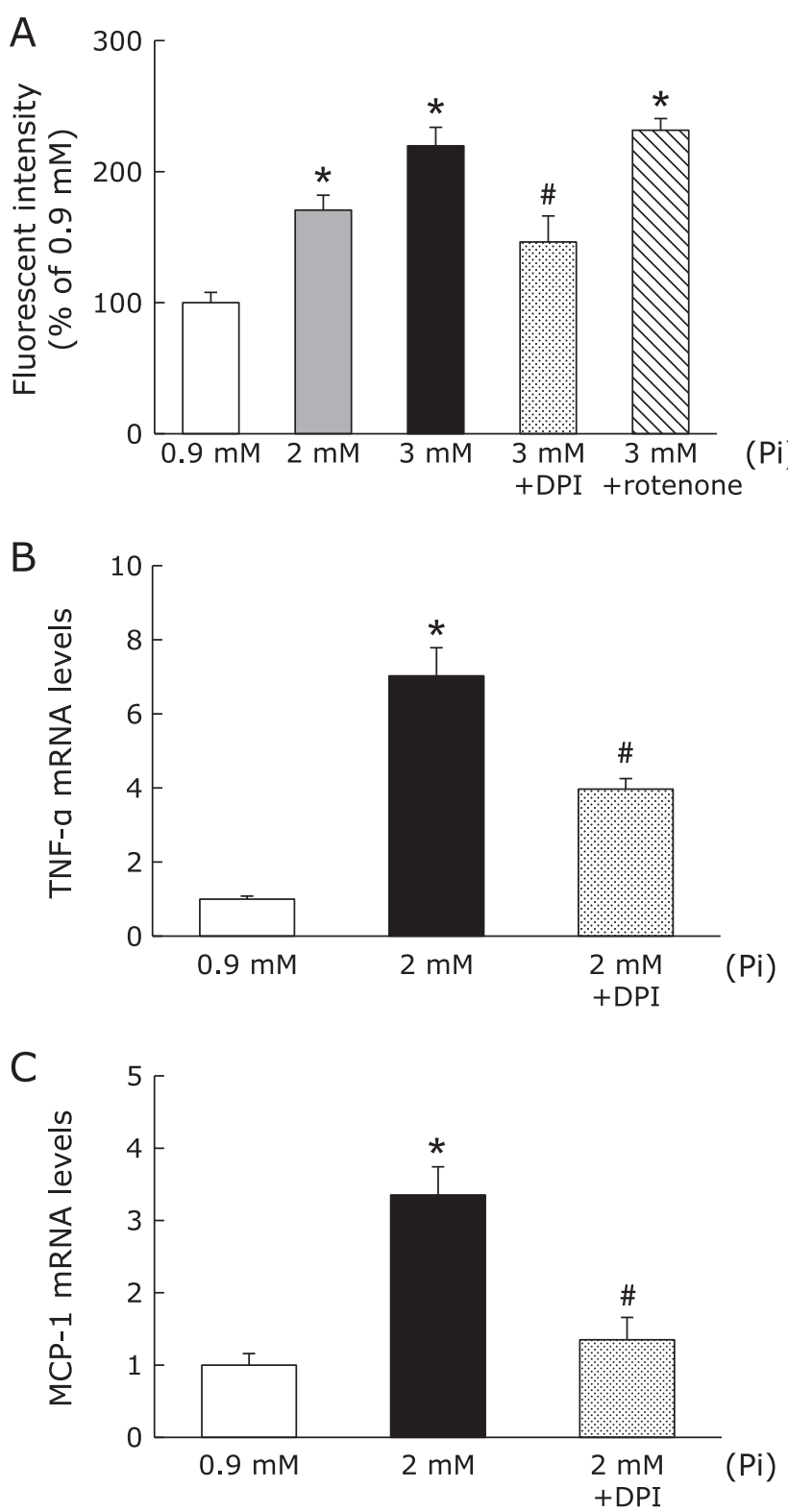

Fig. 7. High Pi loading enhanced ROS production in RAW264.7 cells (A) After incubation with APF $(20 \mu \mathrm{M})$ for 30 min, cells were treated by different concentrations of $\mathrm{Pi}$ for $2 \mathrm{~h}$ with or without various oxidase inhibitors. (B, C) RAW264.7 cells were pretreated with DPI (10 $\mu \mathrm{M})$ for $30 \mathrm{~min}$, and then treated with Pi $(2 \mathrm{mM})$ for $1 \mathrm{~h}$. Data are expressed as mean $\pm \operatorname{SEM}(n=5)$. * $p<0.05$ vs Pi: $0.9 \mathrm{mM}$; ${ }^{*} p<0.05$ vs Pi: $2 \mathrm{mM}$.

thermore, we examined whether inhibition of ROS production could suppress the inflammatory response in RAW264.7 cells. Pretreatment of DPI followed by Pi loading significantly inhibited Pi-induced TNF- $\alpha$ and MCP-1 mRNA expression (Fig. 7B and C). Therefore, Pi-mediated ROS generation could contribute to proinflammatory cytokine/chemokine induction in RAW264.7 cells.

\section{Discussion}

To the best of our knowledge, the present study is the first to demonstrate that high Pi intake exacerbated intestinal inflammation in experimental colitis and high Pi loading increased proinflammatory cytokine expression through ROS production in RAW264.7 macrophages. Furthermore, previous epidemiologic, animal, and clinical studies showed that various nutrients could promote or prevent intestinal inflammation. ${ }^{(2)}$ Indeed, our findings suggest that excessive dietary $\mathrm{Pi}$ is one of the nutrients promoting intestinal inflammation.

Recent widespread consumption of Western diets and processing foods is associated with excessive intake of $\mathrm{Pi}^{\left({ }^{(6-8)}\right.}$ Moreover, previous studies reported that consumption of high $\mathrm{Pi}$ foods (e.g., such as fast foods and processed meat) increased the onset and relapse risk for IBD. ${ }^{(3-5)}$ However, it remains unclear whether dietary $\mathrm{Pi}$ intake is directly correlated to IBD, as the available databases used to estimate dietary $\mathrm{Pi}$ intake are inaccurate, and thus the Pi content of foods is significantly underestimated. ${ }^{(8)}$ Therefore, the present study was performed to examine the impact of dietary Pi on DSS-induced experimental colitis, which mimics ulcerative colitis-like disease. After DSS administration, intestinal inflammation with increased histological scores, NF- $\kappa B$ activation, and proinflammatory cytokine levels were observed and dietary Pi enhanced intestinal inflammation in a dose-dependent manner. Moreover, $\mathrm{Pi}$ acts as an important mediator that stimulates proinflammatory cytokine/chemokine in RAW264.7 macrophages, supporting the hypothesis that tissue resident and recruiting macrophages are the major cell types responsible for adverse effects of Pi on intestinal inflammation.

Several sodium-dependent Pi transporters and Pi uptake were observed in RAW264.7 cells, ${ }^{(30,31)}$ and it is presumed that Pi uptake into RAW264.7 cells results in the activation of macrophages. Oxidative stress has been linked to the pathogenesis of various diseases including IBD. ${ }^{(32)}$ Our study demonstrated that high Pi loading directly enhanced ROS production in RAW264.7 cells. In addition, inhibition of ROS generation using inhibitor of NAD(P)H oxidase partially suppressed Pi-induced inflammatory response. Therefore, Pi-induced ROS production is a possible reason for the activation of RAW264.7 macrophages. In contrast to NAD(P)H oxidase, mitochondrial ROS may only play a partial role in activation of RAW264.7 macrophages. Our results agreed with previous reports by ourselves and others showing that $\mathrm{Pi}$ loading increased ROS production, thus leading to calcification and endothelial dysfunction in a number of cell types. ${ }^{(11-15)}$

$\mathrm{Pi}$ homeostasis is tightly regulated by controlling intestinal and renal epithelial transport systems. Dietary $\mathrm{Pi}$ is absorbed in the proximal small intestine by subtype of type IIb sodium dependent cotransporters (NaPi-IIb) expressed on the apical membranes of enterocytes; an estimated $70 \%$ of all dietary Pi content is absorbed via the small intestine. ${ }^{(33,34)}$ This Pi transport was regulated by various factors, such as Pi intake, ${ }^{(35)}$ vitamin $\mathrm{D}_{3},{ }^{(36)}$ and hormones. ${ }^{(37,38)}$. In addition to these factors, intestinal Pi transport is also affected by intestinal inflammation. Chen et al. ${ }^{(39)}$ reported that intestinal $\mathrm{Pi}$ absorption and $\mathrm{NaPi}-\mathrm{IIb}$ expression were decreased in an experimental colitis model, and they were regulated by TNF- $\alpha$. Therefore, high Pi intake and reduction of intestinal Pi absorption may increase luminal Pi concentrations in the colon. It is widely recognized that the small intestine is responsible for most $\mathrm{Pi}$ absorption, but Pi transport is also observed in the colon. ${ }^{(34,40,41)}$ Although the exact mechanism remains unclear, increased colonic $\mathrm{Pi}$ absorption may accelerate DSS-induced colonic inflammation.

In contrast, serum Pi levels were independently associated with inflammatory markers in CKD patients, ${ }^{(19)}$ and low-Pi diets could ameliorate systemic and renal inflammation in a CKD rat model. ${ }^{(14,42)}$ Moreover, recent studies demonstrated that elevated parathyroid hormone and fibroblast growth factor 23, known as phosphaturic hormones, ${ }^{(6)}$ can directly stimulate hepatic production of inflammatory cytokines, ${ }^{(43,44)}$ and these were positively correlated to inflammatory markers in CKD patients. ${ }^{(45,46)}$ Therefore, we speculate that a high luminal Pi concentration in the colon may enhance colonic inflammation, whereas high $\mathrm{Pi}$ intake-induced systemic alteration in phosphate-regulating hormones can also affect sensitivity to DSS-induced colitis. Since we could not determine which factors are important for intestinal 
inflammation, further investigation is required.

In clinical practice, the harmful effects of Pi should be taken into account. Colonoscopy establishes the diagnosis and subsequent follow-up plan in IBD patients, and oral sodium Pi is widely used as a colonic cleansing agent for colonoscopy. However, oral sodium Pi use may be associated with development of colonic mucosal abnormalities due to acute Pi overloading. ${ }^{(47,48)}$ Therefore, sodium Pi solutions should probably not be used in IBD patients due to the high risk of toxicity on the intestine. Importantly, these agents contain large amounts of $\mathrm{Pi}$ (more than 10 times amount the usual daily Pi dietary intake). On the other hand, the $0.5 \%$ Pi diet was designated as the control in this study and high Pi diets contain more than two or three times that amount. Therefore, a high Pi diet can enhance intestinal inflammation within the range that can be routinely ingested. Furthermore, in terms of therapeutic strategies, excessive $\mathrm{Pi}$ intake should be avoided and a low Pi diet may be beneficial to suppress intestinal inflammation. Since disease activity is correlated with nutritional status, ${ }^{(49)}$ dietary management including phosphate intake is also necessary for improvement of inflammatory status in IBD patients.

In conclusion, our results provide new insights into a dietary

\section{References}

1 Lee D, Albenberg L, Compher C, et al. Diet in the pathogenesis and treatment of inflammatory bowel diseases. Gastroenterology 2015; 148: 1087-1106.

2 Spooren CE, Pierik MJ, Zeegers MP, Feskens EJ, Masclee AA, Jonkers DM. Review article: the association of diet with onset and relapse in patients with inflammatory bowel disease. Aliment Pharmacol Ther 2013; 38: 1172 1187.

3 Persson PG, Ahlbom A, Hellers G. Diet and inflammatory bowel disease: a case-control study. Epidemiology 1992; 3: 47-52.

4 Jowett SL, Seal CJ, Pearce MS, et al. Influence of dietary factors on the clinical course of ulcerative colitis: a prospective cohort study. Gut 2004; 53: 1479-1484.

5 D'Souza S, Levy E, Mack D, et al. Dietary patterns and risk for Crohn's disease in children. Inflamm Bowel Dis 2008; 14: 367-373.

6 Takeda E, Yamamoto H, Yamanaka-Okumura H, Taketani Y. Increasing dietary phosphorus intake from food additives: potential for negative impact on bone health. Adv Nutr 2014; 5: 92-97.

7 Kalantar-Zadeh K, Gutekunst L, Mehrotra R, et al. Understanding sources of dietary phosphorus in the treatment of patients with chronic kidney disease. Clin J Am Soc Nephrol 2010; 5: 519-530.

8 Calvo MS, Uribarri J. Public health impact of dietary phosphorus excess on bone and cardiovascular health in the general population. Am J Clin Nutr 2013; 98: 6-15.

9 Yamamoto KT, Robinson-Cohen C, de Oliveira MC, et al. Dietary phosphorus is associated with greater left ventricular mass. Kidney Int 2013; 83: 707-714.

10 Chang AR, Lazo M, Appel LJ, Gutiérrez OM, Grams ME. High dietary phosphorus intake is associated with all-cause mortality: results from NHANES III. Am J Clin Nutr 2014; 99: 320-327.

11 Shuto E, Taketani Y, Tanaka R, et al. Dietary phosphorus acutely impairs endothelial function. J Am Soc Nephrol 2009; 20: 1504-1512.

12 Di Marco GS, Hausberg M, Hillebrand U, et al. Increased inorganic phosphate induces human endothelial cell apoptosis in vitro. Am J Physiol Ren Physiol 2008; 294: F1381-F1387.

13 Nguyen TT, Quan X, Hwang KH, et al. Mitochondrial oxidative stress mediates high phosphate-induced secretory defects and apoptosis in insulinsecreting cells. Am J Physiol Endocrinol Metab 2015; 308: E933-E941.

14 Yamada S, Tokumoto M, Tatsumoto N, et al. Phosphate overload directly induces systemic inflammation and malnutrition as well as vascular calcification in uremia. Am J Physiol Ren Physiol 2014; 306: F1418-F1428.

15 Zhao MM, Xu MJ, Cai Y, et al. Mitochondrial reactive oxygen species promote p65 nuclear translocation mediating high-phosphate-induced vascular calcification in vitro and in vivo. Kidney Int 2011; 79: 1071-1079.

16 Adeney KL, Siscovick DS, Ix JH, et al. Association of serum phosphate with vascular and valvular calcification in moderate CKD. J Am Soc Nephrol 2009; 20: 381-387. therapeutic strategy for IBD based on observations that excessive dietary Pi exacerbated intestinal inflammation through the production of proinflammatory cytokines/chemokines from activated macrophages. Further epidemiological and clinical studies are required to clarify how dietary $\mathrm{Pi}$ is directly associated with pathogenesis and the clinical condition of IBD patients.

\section{Acknowledgments}

We sincerely thank Prof. Takeshi Nikawa and Dr. Tomoki Abe (Tokushima University, Tokushima, Japan) for kindly providing us with RAW264.7 cells and BZ-9000 fluorescence microscope. We also thank M. Sogo, S. Fukuda, Y. Ishida, N. Yabiki, A. Takeuchi, S. Sakai (Tokushima University Graduate School, Tokushima, Japan) for technical assistance. This work was supported by the Grants-in-Aid for Scientific Research (B) (16H03046 for YT and 25282022 for HY) from Japan Society for the Promotion of Science (JSPS).

\section{Conflict of Interest}

No potential conflicts of interest were disclosed.

17 Block GA, Klassen PS, Lazarus JM, Ofsthun N, Lowrie EG, Chertow GM. Mineral metabolism, mortality, and morbidity in maintenance hemodialysis. $J$ Am Soc Nephrol 2004; 15: 2208-2218.

18 Ikeda S, Yamamoto H, Masuda M, et al. Downregulation of renal type IIa sodium-dependent phosphate cotransporter during lipopolysaccharide-induced acute inflammation. Am J Physiol Ren Physiol 2014; 306: F744-F750.

19 Navarro-González JF, Mora-Fernández C, Muros M, Herrera H, García J. Mineral metabolism and inflammation in chronic kidney disease patients: a cross-sectional study. Clin J Am Soc Nephrol 2009; 4: 1646-1654.

20 Nakao M, Yamamoto H, Nakahashi O, et al. Dietary phosphate supplementation delays the onset of iron deficiency anemia and affects iron status in rats. Nutr Res 2015; 35: 1016-1024.

21 Abuduli M, Ohminami H, Otani T, et al. Effects of dietary phosphate on glucose and lipid metabolism. Am J Physiol Endocrinol Metab 2016; 310: E526-E538.

22 Cooper HS, Murthy SN, Shah RS, Sedergran DJ. Clinicopathologic study of dextran sulfate sodium experimental murine colitis. Lab Invest 1993; 69: 238-249.

23 Vowinkel T, Mori M, Krieglstein CF, et al. Apolipoprotein A-IV inhibits experimental colitis. J Clin Invest 2004; 114: 260-269.

24 Krawisz JE, Sharon P, Stenson WF. Quantitative assay for acute intestinal inflammation based on myeloperoxidase activity. Assessment of inflammation in rat and hamster models. Gastroenterology 1984; 87: 1344-1350.

25 Viennois E, Chen F, Laroui H, Baker MT, Merlin D. Dextran sodium sulfate inhibits the activities of both polymerase and reverse transcriptase: lithium chloride purification, a rapid and efficient technique to purify RNA. BMC Res Notes 2013; 6: 360.

26 Masuda M, Yamamoto H, Kozai M, et al. Regulation of renal sodiumdependent phosphate co-transporter genes (Npt2a and Npt2c) by all-transretinoic acid and its receptors. Biochem J 2010; 429: 583-592.

27 Setsukinai K, Urano Y, Kakinuma K, Majima HJ, Nagano T. Development of novel fluorescence probes that can reliably detect reactive oxygen species and distinguish specific species. J Biol Chem 2003; 278: 3170-3175.

28 Wagner AE, Boesch-Saadatmandi C, Dose J, Schultheiss G, Rimbach G. Anti-inflammatory potential of allyl-isothiocyanate--role of Nrf2, NF- $\kappa \mathrm{B}$ and microRNA-155. J Cell Mol Med 2012; 16: 836-843.

29 Morgan MJ, Liu ZG. Crosstalk of reactive oxygen species and NF- $\kappa$ B signaling. Cell Res 2011; 21: 103-115.

30 Ito M, Matsuka N, Izuka M, et al. Characterization of inorganic phosphate transport in osteoclast-like cells. Am J Physiol Cell Physiol 2005; 288: C921C931.

31 Albano G, Moor M, Dolder S, et al. Sodium-dependent phosphate transporters in osteoclast differentiation and function. PLoS One 2015; 10: e0125104. 
32 Rezaie A, Parker RD, Abdollahi M. Oxidative stress and pathogenesis of inflammatory bowel disease: an epiphenomenon or the cause? Dig Dis Sci 2007; 52: 2015-2021.

33 Sabbagh Y, O'Brien SP, Song W, et al. Intestinal npt $2 b$ plays a major role in phosphate absorption and homeostasis. J Am Soc Nephrol 2009; 20: 23482358.

34 Marks J, Lee GJ, Nadaraja SP, Debnam ES, Unwin RJ. Experimental and regional variations in $\mathrm{Na}+$-dependent and $\mathrm{Na}+$-independent phosphate transport along the rat small intestine and colon. Physiol Rep 2015; 3: e12281.

35 Hattenhauer O, Traebert M, Murer H, Biber J. Regulation of small intestinal Na-P(i) type IIb cotransporter by dietary phosphate intake. Am J Physiol 1999; 277 (4 Pt 1): G756-G762.

$36 \mathrm{Xu} \mathrm{H}$, Bai L, Collins JF, Ghishan FK. Age-dependent regulation of rat intestinal type IIb sodium-phosphate cotransporter by $1,25-(\mathrm{OH})(2)$ vitamin D(3). Am J Physiol Cell Physiol 2002; 282: C487-C493.

37 Arima K, Hines ER, Kiela PR, Drees JB, Collins JF, Ghishan FK. Glucocorticoid regulation and glycosylation of mouse intestinal type IIb Na-P(i) cotransporter during ontogeny. Am J Physiol Gastrointest Liver Physiol 2002; 283: G426-G434.

$38 \mathrm{Xu} \mathrm{H}$, Uno JK, Inouye $\mathrm{M}$, et al. Regulation of intestinal NaPi-IIb cotransporter gene expression by estrogen. Am J Physiol Gastrointest Liver Physiol 2003; 285: G1317-G1324.

39 Chen H, Xu H, Dong J, Li J, Ghishan FK. Tumor necrosis factor-alpha impairs intestinal phosphate absorption in colitis. Am J Physiol Gastrointest Liver Physiol 2009; 296: G775-G781.

40 Hu MS, Kayne LH, Jamgotchian N, Ward HJ, Lee DB. Paracellular phosphate absorption in rat colon: a mechanism for enema-induced hyperphosphatemia. Miner Electrolyte Metab 1997; 23: 7-12.
41 Hilfiker H, Hattenhauer O, Traebert M, Foster I, Murer H, Biber J. Characterization of a murine type II sodium-phosphate cotransporter expressed in mammalian small intestine. Proc Natl Acad Sci U S A 1998; 95: 14564 14569.

42 Finch JL, Lee DH, Liapis H, et al. Phosphate restriction significantly reduces mortality in uremic rats with established vascular calcification. Kidney Int 2013; 84: 1145-1153.

43 Mitnick MA, Grey A, Masiukiewicz U, et al. Parathyroid hormone induces hepatic production of bioactive interleukin-6 and its soluble receptor. Am J Physiol Endocrinol Metab 2001; 280: E405-E412.

44 Singh S, Grabner A, Yanucil C, et al. Fibroblast growth factor 23 directly targets hepatocytes to promote inflammation in chronic kidney disease. Kidney Int 2016; 90: 985-996.

45 Cheng SP, Liu CL, Liu TP, Hsu YC, Lee JJ. Association between parathyroid hormone levels and inflammatory markers among US adults. Mediators Inflamm 2014; 2014: 709024.

46 Munoz Mendoza J, Isakova T, Ricardo AC, et al. Fibroblast growth factor 23 and Inflammation in CKD. Clin J Am Soc Nephrol 2012; 7: 1155-1162.

47 Zwas FR, Cirillo NW, el-Serag HB, Eisen RN. Colonic mucosal abnormalities associated with oral sodium phosphate solution. Gastrointest Endosc 1996; 43: 463-466.

48 Coskun A, Uzunkoy A, Duzgun SA, Bozer M, Ozardali I, Vural H. Experimental sodium phosphate and polyethylene glycol induce colonic tissue damage and oxidative stress. Br J Surg 2001; 88: 85-89.

49 Takaoka A, Sasaki M, Kurihara M, et al. Comparison of energy metabolism and nutritional status of hospitalized patients with Crohn's disease and those with ulcerative colitis. J Clin Biochem Nutr 2015; 56: 208-214. 